ARTICLE

\title{
Smoothing the energy transfer pathway in quasi- 2D perovskite films using methanesulfonate leads to highly efficient light-emitting devices
}

\author{
Lingmei Kong (10 1,8, Xiaoyu Zhang (10) 2,8, Yunguo Li (10 ${ }^{3,8}$, Haoran Wang${ }^{1}$, Yuanzhi Jiang (1) 4, Sheng Wang ${ }^{1}$, \\ Mengqing You ${ }^{1}$, Chengxi Zhang (1) ${ }^{1}$, Ting Zhang ${ }^{1}$, Stephen V. Kershaw ${ }^{5}$, Weitao Zheng ${ }^{2}$, Yingguo Yang (1) ${ }^{6}$, \\ Qianqian Lin (1) ${ }^{7}$, Mingjian Yuan ${ }^{4}$, Andrey L. Rogach (i) ${ }^{5 凶} \&$ Xuyong Yang (iD) ${ }^{1 凶}$
}

Quasi-two-dimensional (quasi-2D) Ruddlesden-Popper (RP) perovskites such as $\mathrm{BA}_{2} \mathrm{Cs}_{n-1} \mathrm{~Pb}_{n} \mathrm{Br}_{3 n+1}(\mathrm{BA}=$ butylammonium, $n>1)$ are promising emitters, but their electroluminescence performance is limited by a severe non-radiative recombination during the energy transfer process. Here, we make use of methanesulfonate (MeS) that can interact with the spacer BA cations via strong hydrogen bonding interaction to reconstruct the quasi-2D perovskite structure, which increases the energy acceptor-to-donor ratio and enhances the energy transfer in perovskite films, thus improving the light emission efficiency. MeS additives also lower the defect density in RP perovskites, which is due to the elimination of uncoordinated $\mathrm{Pb}^{2}+$ by the electron-rich Lewis base MeS and the weakened adsorbate blocking effect. As a result, green light-emitting diodes fabricated using these quasi-2D RP perovskite films reach current efficiency of $63 \mathrm{~cd} \mathrm{~A}^{-1}$ and 20.5\% external quantum efficiency, which are the best reported performance for devices based on quasi-2D perovskites so far.

\footnotetext{
${ }^{1}$ Key Laboratory of Advanced Display and System Applications of Ministry of Education, Shanghai University, Shanghai 200072, People's Republic of China. ${ }^{2}$ College of Materials Science and Engineering, Jilin University, Changchun 130012, People's Republic of China. ${ }^{3}$ CAS Key Laboratory of Crust-Mantle Materials and Environments, School of Earth and Space Sciences, University of Science and Technology of China, Hefei 230026, People's Republic of China. ${ }^{4}$ Key Laboratory of Advanced Energy Materials Chemistry (Ministry of Education), Renewable Energy Conversion and Storage Center (RECAST), College of Chemistry, Nankai University, Tianjin 300071, People's Republic of China. ${ }^{5}$ Department of Materials Science and Engineering, and Centre for Functional Photonics (CFP), City University of Hong Kong, Kowloon, Hong Kong SAR, People's Republic of China. ${ }^{6}$ Shanghai Synchrotron Radiation Facility (SSRF), Zhangjiang Lab, Shanghai Advanced Research Institute, Chinese Academy of Sciences, Shanghai 201204, People's Republic of China. ${ }^{7}$ Key Lab of Artificial Micro- and Nano-Structures of Ministry of Education of China, School of Physics and Technology, Wuhan University, Wuhan 430072, People's Republic of China. ${ }^{8}$ These authors contributed equally: Lingmei Kong, Xiaoyu Zhang, Yunguo Li. ${ }^{凶}$ email: andrey.rogach@cityu.edu.hk; yangxy@shu.edu.cn
} 
M etal halide perovskites exhibit distinctive properties such as tunable bandgaps and narrow emission linewidths, which are combined with low cost and facile solution processability ${ }^{1-10}$. These useful characteristics have made them extremely promising candidates for light-emitting diode (LED) and display applications ${ }^{11}$. Tremendous progress has already been achieved in improving perovskite LEDs (PeLEDs) based on threedimensional (3D) perovskite films $\mathrm{s}^{2,12-17}$, but their overall electroluminescence (EL) performance still lags behind those of state-ofthe-art organic LEDs and other solid-state lighting devices such as inorganic quantum-dot-based LEDs ${ }^{11,18-20}$. A slow electron-hole capture rate stemming from inherent small exciton binding (only tens of $\mathrm{meV}$ ) and long diffusion length leads to inefficient radiative recombination in 3D perovskites ${ }^{21-24}$, going against high emission efficiency naturally.

Quasi-two-dimensional (quasi-2D) perovskites offer larger exciton binding energy (hundreds of $\mathrm{meV}$ ) and lower ion mobility compared with $3 \mathrm{D}$ perovskites ${ }^{23,25-28}$, making them even more promising light-emitting materials. Quasi-2D perovskites with Ruddlesden-Popper (RP) structure have the general formula of $\mathrm{L}_{2} \mathrm{~A}_{n-1} \mathrm{M}_{n} \mathrm{X}_{3 n+1}{ }^{23,24,29}$, where $\mathrm{L}$ is a large-size organic spacer cation with low conductivity ("barrier") such as butylammonium (BA), phenylethylammonium (PEA), naphthylmethylamine (NMA), etc., A is Cs, methylammonium (MA), or formamidinium (FA), $\mathrm{M}$ is a divalent metal cation such as $\mathrm{Pb}$ or $\mathrm{Sn}, \mathrm{X}$ is a halide anion $(\mathrm{Cl}, \mathrm{Br}$, or $\mathrm{I})$, and $n$ is the number of $\mathrm{MX}_{6}^{4-}$ sheets (which constitute a "quantum well", QW) sandwiched between the organic spacers ${ }^{25}$. There are two key points that determine the emission efficiency of quasi-2D RP perovskites, namely the exciton energy transfer process and the deep defect state formation derived from the bandgap broadening ${ }^{30-32}$. The distribution of energy domains is inhomogeneous in quasi-2D perovskites due to the random stacking of low-dimensional structures with varying degrees of quantum and dielectric confinement $6,33,34$. The small- $n$ phases $(n \leq 3)$ with wide-bandgap QWs are prone to form because of their low formation energy ${ }^{35}$, resulting in inefficient energy transfer processes from small- $n$ phases to large- $n$ phases. The defect states that result from lattice defects and the bandgap broadening due to the quantum confinement effect, act as charge-carrier traps during the energy transfer process, which lowers the emission efficiency.

Here, we seek to realize the full potential of quasi-2D perovskites for LEDs by smoothing the energy transfer pathway through structure reconstruction and defect reduction using methanesulfonate (MeS) additive. The strong hydrogen-bonding interaction between $\mathrm{MeS}$ and BA spacer cations effectively modulates the crystallization kinetics and leads to an increased ratio of large- $n$ phase with narrow bandgap QWs, while the uncoordinated $\mathrm{Pb}^{2+}$ ions on the perovskite surface are simultaneously reduced due to the weakened adsorbate blocking effect caused by MeS addition, as well as the formation of Lewis adducts. Quasi-2D green PeLEDs fabricated following this strategy show current efficiency (CE) of $63 \mathrm{~cd} \mathrm{~A}^{-1}$ and up to $20.5 \%$ external quantum efficiency (EQE), making this device the bestperforming quasi-2D perovskite LED reported in literature so far.

\section{Results}

Fabrication of MeS-treated $\mathrm{BA}_{2} \mathrm{Cs}_{n-1} \mathrm{~Pb}_{n} \mathrm{Br}_{3 n+1}$ perovskite films. The perovskite precursor solutions comprised of cesium bromide ( $\mathrm{CsBr})$, lead bromide $\left(\mathrm{PbBr}_{2}\right)$, butylammonium bromide (BABr), poly(ethylene oxide) (PEO), and a varying ratio of cesium methanesulfonate (CsMeS) in dimethyl sulfoxide (DMSO) (see Methods for details). Here, the ratio of the added MeS is defined as the molar ratio of CsMeS-to- $\mathrm{PbBr}_{2}$ (denoted as " $x \%$-MeS" further on). The MeS-treated quasi-2D perovskite films with a chemical formula of $\mathrm{BA}_{2} \mathrm{Cs}_{n-1} \mathrm{~Pb}_{n} \mathrm{Br}_{3 n+1}$ were prepared on indium tin oxide (ITO) substrates pre-coated with poly(9,9-dioctylfluorenyl-2,7-diyl)co-(4,4'-(N-(4-sec-butylphenyl)diphenylamine) (TFB): poly(9-vinylcarbazole) (PVK) hole transport layer (HTL) and polyvinylpyrrolidone (PVP) interface modified layer by one-step solution coating of the precursor solutions, followed by thermal annealing under nitrogen atmosphere, as schematically illustrated in Fig. 1. The

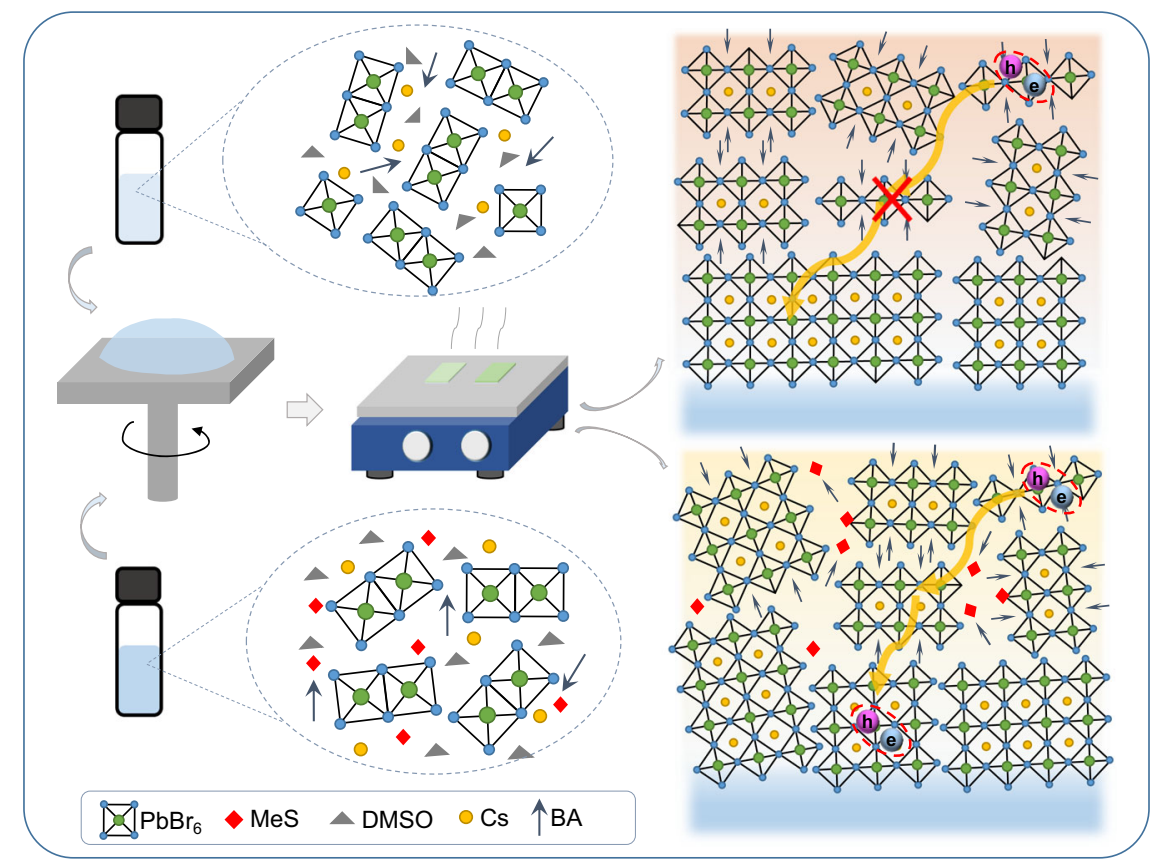

Fig. 1 Effects of $\mathbf{M e S}$ on phase distribution of $\mathbf{B A}_{\mathbf{2}} \mathbf{C s}_{n-1} \mathbf{P b}_{n} \mathbf{B r}_{3 n+1}$ perovskite films. Schematic illustrations of the colloidal clusters' structures in precursor solutions and phase distributions of $n$ values in films after thermal annealing without (top pathway) and with (bottom pathway) addition of MeS, where different energy transfer pathways from small-n phases to large- $n$ phases are presented. Crystal orientations in the perovskite films were verified by grazing-incidence wide-angle X-ray scattering (GIWAXS) measurements (see Supplementary Fig. 2). 
CsMeS molecule consists of two parts: a $\mathrm{Cs}^{+}$cation and a $\mathrm{MeS}^{-}$ anion, whose molecular structure is shown in Supplementary Fig. 1. The introduction of $\mathrm{MeS}$ in precursor solution can control the crystallization growth kinetics of quasi-2D perovskite films resulting in enhanced exciton energy transfer from small- $n$ phases to large- $n$ phases, which will be discussed below in detail.

Effect of MeS addition on growth of quasi-2D perovskite films. Density functional theory (DFT) calculations (see Methods) reveal that the spacer $\mathrm{BA}$ prefers to bind $\mathrm{MeS}$ via three hydrogen bonds rather than to bind $\mathrm{Br}$ anion. The following reaction was investigated by DFT simulations:

$$
\mathrm{BABr}+\mathrm{CsMeS}=\mathrm{BAMeS}+\mathrm{CsBr}
$$

where $\mathrm{BABr}, \mathrm{CsMeS}$, and $\mathrm{BAMeS}$ were considered in the form of free molecules, and $\mathrm{CsBr}$ in the crystalline form of space group $F m \overline{3} m$. Their relaxed structures are shown in Supplementary Fig. 1. The calculated reaction free energy is $-2.16 \mathrm{eV}$, indicating a strong driving force toward the formation of BAMeS. Indeed, the binding energy of $\mathrm{BA}$ and $\mathrm{MeS}$ was calculated to be $4.96 \mathrm{eV}$, which is much higher than both of $\mathrm{BA}$ and $\mathrm{Br}(4.00 \mathrm{eV})$, and $\mathrm{Cs}$ and $\mathrm{MeS}(4.39 \mathrm{eV})$. This suggests that $\mathrm{MeS}$ tends to bind $\mathrm{BA}$ via triple hydrogen bonding. The formation of hydrogen bonding incurs a charge redistribution of 0.84 e (electron) between BA and $\mathrm{MeS}$, which is larger than that $(0.70 \mathrm{e})$ between $\mathrm{BA}$ and $\mathrm{Br}$ (Fig. 2). Therefore, the strong affinity of the spacer cation for MeS fosters a higher probability for spacer-free nanosheet formation, and their stacking growth. This interaction is further confirmed by ${ }^{1} \mathrm{H}$ nuclear magnetic resonance (NMR) spectra (Supplementary Fig. 3). The proton resonance signal of ammonium $\left(\mathrm{NH}_{3}{ }^{+}\right)$ of BA (peak at $\delta=7.65 \mathrm{ppm}$ ) shifts to low-field after incorporation of $\mathrm{MeS}(7.70 \mathrm{ppm})$. The variation in the chemical shift indicates the formation of hydrogen bonds between BA and MeS, which leads to the deshielding effect ${ }^{36,37}$. This enables us to increase the proportion of large- $n$ phases in the overall perovskite phase distribution.
$\mathrm{BABr}$

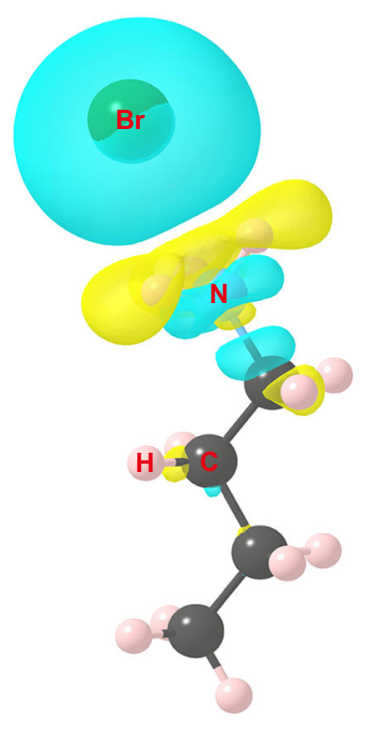

Total charge transfer $0.70 \mathrm{e}$
BAMeS

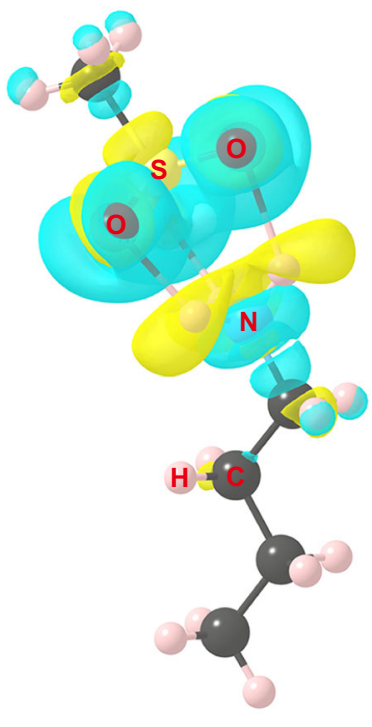

Total charge transfer $0.84 \mathrm{e}$
Fig. 2 Hydrogen bonding calculations. Differential charge density plots (isosurface value of $0.0015 \mathrm{eV} \AA-3$; cyan, charge accumulation; yellow, charge depletion) of $\mathrm{BABr}$ and $\mathrm{BAMeS}$ show that the bonding between $\mathrm{BA}$ and $\mathrm{MeS}$ induces more charge redistribution than that between $\mathrm{BA}$ and $\mathrm{Br}$.
MeS molecules are homogeneously distributed over the quasi2D perovskite films as shown in the energy-dispersive X-ray (EDX) mapping data (Supplementary Fig. 4). To address the influence of $\mathrm{MeS}$ on the perovskite phase distribution, measurements of the X-ray photoelectron spectra (XPS) that track the $\mathrm{N}$ signal (where BA is the only source of that atomic species and thus the signal stands for the phase distribution changes) along with decreasing the perovskite film thickness by increasing the $\mathrm{Ar}^{+}$etching time were conducted. The gradual decrease in $\mathrm{N}$ content for both control and MeS-treated quasi-2D perovskite films were revealed (Supplementary Fig. 5), indicating that the multiple phases existing in quasi-2D perovskite films are arranged from small- $n$ to large- $n$ in the direction perpendicular to the substrate. The S signal (where MeS was the only source of that species) has a gradient content from high to low across the film from the top to the bottom (Supplementary Fig. 5b), resulting in an orderly phase arrangement in the vertical crystal spatial distribution.

Absorption spectra were measured to further analyze the effect of $\mathrm{MeS}$ on the phase distribution of quasi-2D perovskites. The control film produced without $\mathrm{MeS}$ treatment exhibits several exciton absorption peaks located at 403, 434, 467, and $516 \mathrm{~nm}$ (Supplementary Fig. 6), which correspond to phases with $n=1,2,3$, and $n \geq 4$, respectively ${ }^{38-40}$. The MeS-treated film exhibits weaker absorption peaks for small- $n$ phases, and the $10 \%-\mathrm{MeS}$-treated film exhibits only one sharp exciton absorption peak (Supplementary Fig. 7a), suggesting a decrease in the content of small- $n$ phases as the MeS content increased. As shown in the corresponding XRD patterns (Supplementary Fig. 7b, c), the appearance of the (002) plane $^{41}$ for the $n=1$ phase is hindered with increasing MeS content, consistent with the absorption analysis.

Energy transfer in quasi-2D perovskite films. The exciton energy transfer in quasi-2D perovskite films was studied by transient absorption (TA) spectroscopy and steady-state photoluminescence (PL), and time-resolved photoluminescence (TRPL) spectroscopy. TA spectra shown in Fig. 3a-d reveal how the addition of $\mathrm{MeS}$ influences the dynamics of photo-generated carriers within quasi-2D perovskite films. The control perovskite film features four pronounced ground-state bleach (GSB) peaks at $404,436,465$, and $513 \mathrm{~nm}$ corresponding to $n=1,2,3$, and $n \geq 4$ phases in the TA spectra at different selected delay times, respectively (Fig. 3a). The peak positions of these transitions are consistent with those observed in the steady-state absorption spectra (Supplementary Fig. 7a). Note that the exciton resonance at $\mathrm{GSB}_{n=2}$ persists after a long excitation time (101 ps), suggesting that there are excitons accumulated in the $n=2$ phase in particular, resulting from the incomplete energy transfer between the different phases ${ }^{42}$.

For the $8 \%$-MeS-treated perovskite film, the exciton resonance at $\mathrm{GSB}_{n=1}$ does not appear in the TA spectrum (Fig. 3b), indicating that the population fraction of the small- $n$ phases was dramatically reduced. At a probe time of $101 \mathrm{ps,} \mathrm{only} \mathrm{the} \mathrm{exciton}$ bleach at $\mathrm{GSB}_{n \geq 4}$ was observed in this sample. This is further confirmed by visualizing the same data as TA kinetics traces (Fig. 2c, d). The ultrafast decay component for the control film exhibits time constants $\left(\tau_{1}\right)$ of $144 \mathrm{fs}(n=1), 329 \mathrm{fs}(n=2)$, and $973 \mathrm{fs}(n=3)$, which is closely matched with the formation (rise) time constant $\left(\tau_{\text {et }}\right)$ of $1202 \mathrm{fs}$ for $\mathrm{GSB}_{n \geq 4}$ (Supplementary Table 1). In contrast, the decay kinetics for $\mathrm{GSB}_{n \geq 4}$ peak of $8 \%$-MeS-treated perovskite film shows a shorter formation time of $910 \mathrm{fs}$, demonstrating that an accelerated exciton energy transfer occurs inside the reconstructed phases enabling more efficient energy transfer from small- $n$ phases to large- $n$ phases. 


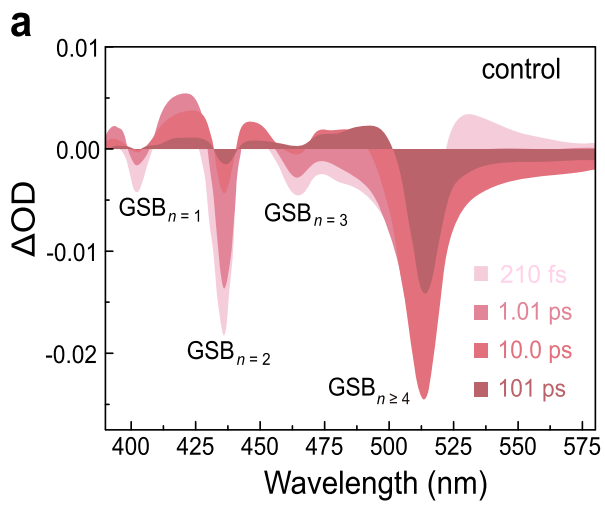

C

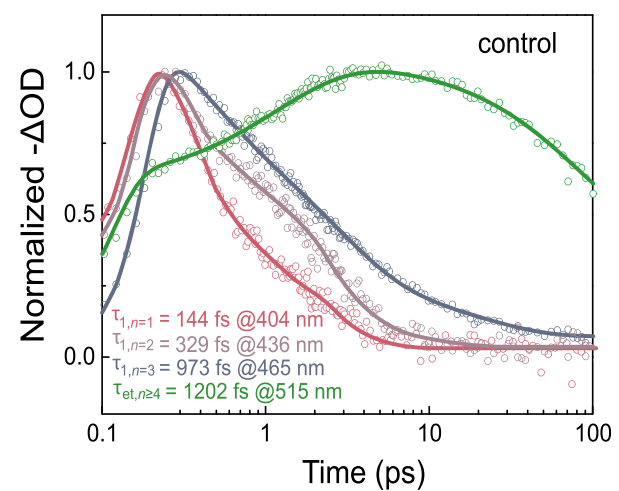

e

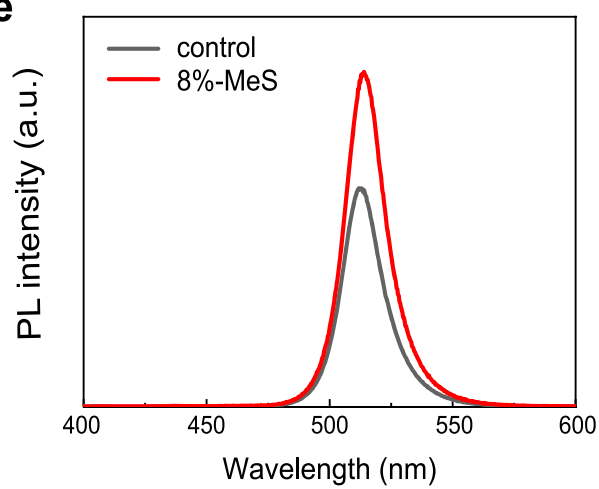

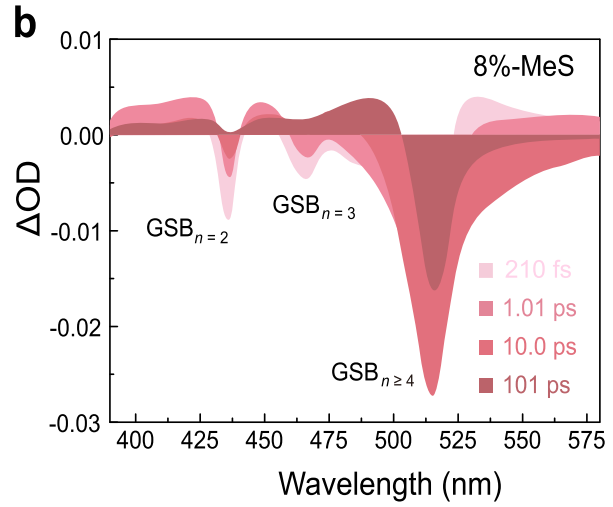

d

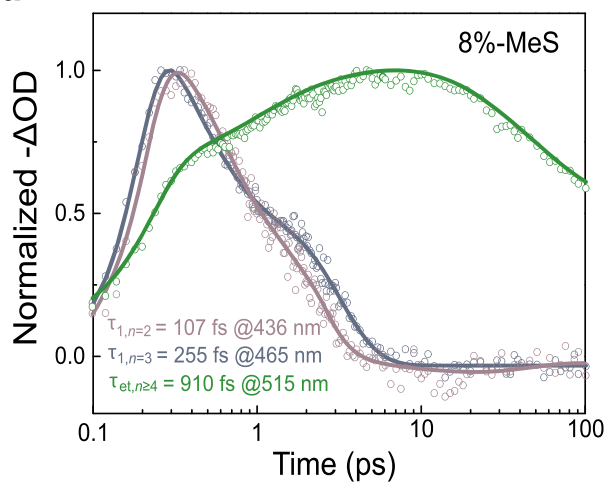

f

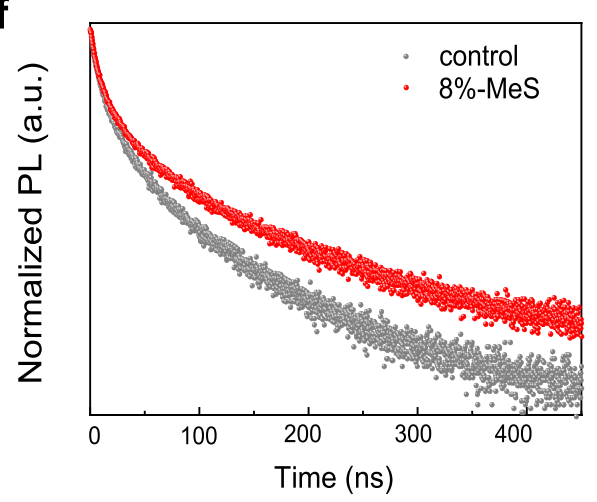

Fig. 3 TA spectra and PL characteristics of quasi-2D perovskite films. a, $\mathbf{b}$ TA spectra at selected timescales. $\mathbf{c}$, $\mathbf{d}$ TA kinetics traces probed at a different wavelength for the control (a, c) and MeS-treated perovskite films (b, d), respectively (excited at $365 \mathrm{~nm}$ ). e Steady-state PL spectra and $\mathbf{f}$ TRPL decay of the control (emission wavelength: $512 \mathrm{~nm}$ ) and MeS-treated perovskite film on glass substrates (emission wavelength: $514 \mathrm{~nm}$ ) excited at $365 \mathrm{~nm}$.

The steady-state PL spectra and TRPL spectroscopy analysis are in good agreement with the results of TA kinetics. The 8\%MeS-treated perovskite film shows a much higher PL quantum yield (PLQY) of 73\% than that of $47 \%$ for the control perovskite film (Fig. 3e and Supplementary Fig. 8). Such a dramatic improvement can be attributed to the improved energy transfer from small- $n$ to large- $n$ phases, ultimately enhancing the radiative recombination in the large- $n$ phases. We also observed a slight redshift of the PL emission upon increasing the MeS content which is caused by the increased proportion of large- $n$ phases ${ }^{24}$. TRPL spectroscopy shows an average recombination lifetime $\left(\tau_{\text {avg }}\right)$ of $101 \mathrm{~ns}$ for $8 \%$-MeS-treated films (Supplementary Table 2), which constitutes a 1.3-fold increase as compared to the control perovskite film (77 ns), corresponding to the enlarged perovskite crystal sizes (shift to higher $n$ ) after MeS treatment. For the control perovskite film, the PLQY was around $47 \%$ which, if the proportion of dark emitters is neglected, would imply almost equal radiative and non-radiative recombination rates The fact that the PLQY increases to $73 \%$ with the MeS-treated perovskite, even though the decay rate is slower, indicates that the effective non-radiative rate must also have significantly slowed down (or the dark fraction of emitters has significantly dropped), which in any case would result in brighter emission (and indeed, both of these factors could have jointly contributed to the improved PLQY). In the further film measurements discussed below, we offer evidence that a significant contribution to the reduction in the non-radiative rate is the decrease of trap densities in MeS-treated perovskite.

Apart from the structural reconstruction of perovskite films, the elimination (or partial elimination) of uncoordinated $\mathrm{Pb}^{2+}$ ions is yet another factor that ensures a more efficient exciton energy transfer pathway. Excitons trapped by uncoordinated $\mathrm{Pb}^{2+}$ are prone to recombine non-radiatively ${ }^{43}$. For the case of the samples discussed here, oxygen $(\mathrm{O})$ atoms possessing lone pair electrons on 

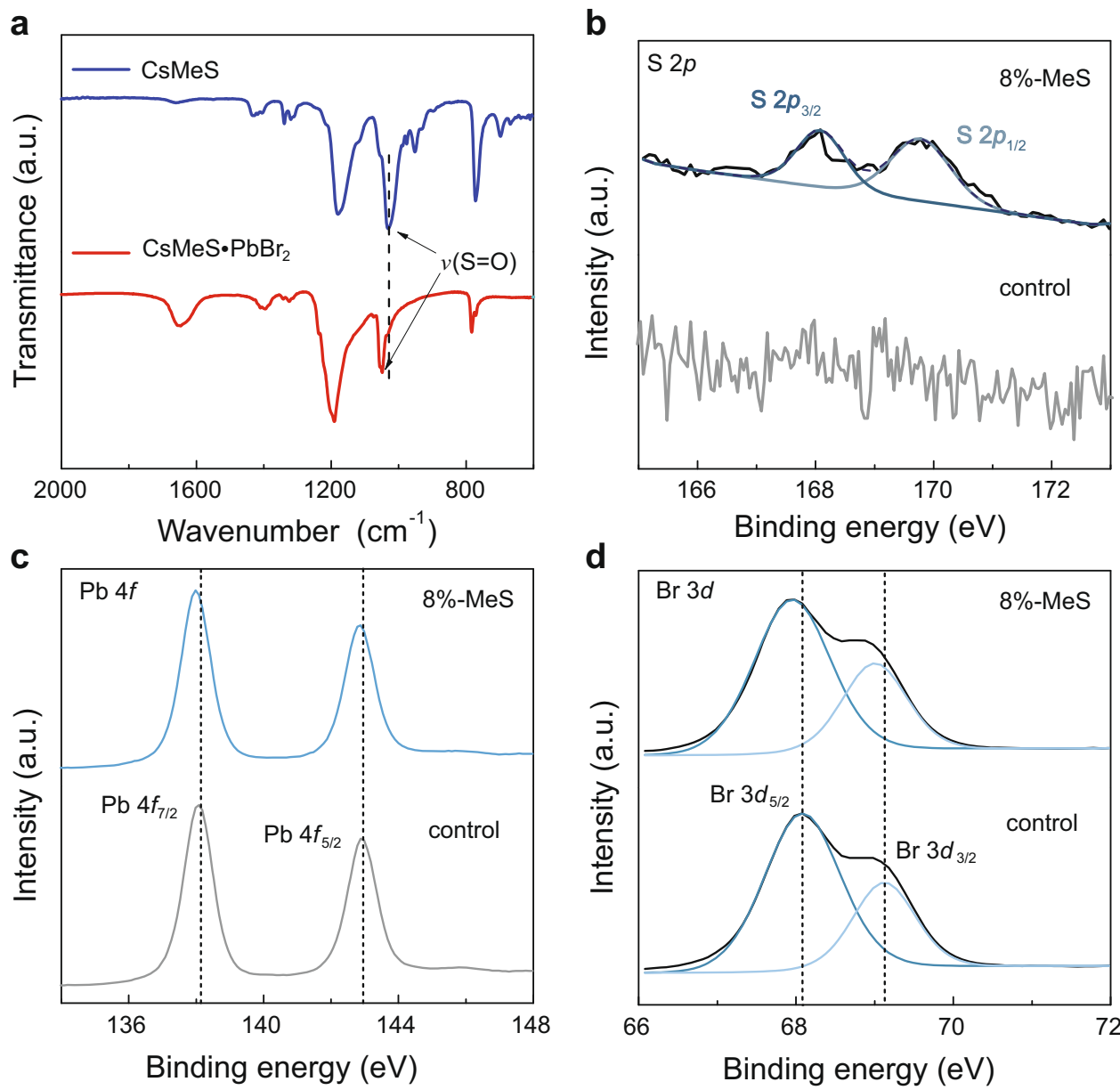

Fig. 4 FTIR and XPS spectra. a FTIR spectra of powdered CsMeS and CsMeS.PbBr ${ }_{2}$, and high-resolution XPS spectra of the control and MeS-treated films for: $\mathbf{b} \mathrm{S} 2 p, \mathbf{c} \mathrm{Pb} 4 f$, and $\mathbf{d} \mathrm{Br} 3 d$.

the $\mathrm{S}=\mathrm{O}$ groups of $\mathrm{MeS}$ could coordinate with the $\mathrm{Pb}$-exposed surface through the formation of Lewis adducts ${ }^{44}$, favoring an efficient energy transfer into the emitting phases (as illustrated in Fig. 3f). Also, the reduction of small- $n$ phases-which would appear to feature a greater abundance of non-radiative trap sitesshould improve the radiative recombination ${ }^{45}$. Evidence of the coordination between $\mathrm{MeS}$ and $\mathrm{Pb}^{2+}$ is obtained from Fourier transform infrared (FTIR) and XPS (Fig. 4). The peak at $1029 \mathrm{~cm}^{-1}$ derived from the $\mathrm{S}=\mathrm{O}$ stretching vibration $(v(\mathrm{~S}=\mathrm{O}))$ of the $-\mathrm{SO}_{3}{ }^{-}$ in FTIR spectra of powdered $\mathrm{CsMeS}^{41}$ shifts to a higher wavenumber of $1052 \mathrm{~cm}^{-1}$ in the presence of $\mathrm{PbBr}_{2}$, indicating the coordination of $\mathrm{MeS}$ and $\mathrm{Pb}^{2+}$ cations ${ }^{46-48}$. To further confirm whether this coordination exists in the MeS-treated perovskite films, we conducted XPS analysis of high-resolution $\mathrm{S} 2 p, \mathrm{~Pb} 4 f$, and $\mathrm{Br} 3 d$ chemical states. As shown in Fig. $4 \mathrm{~b}$, the peaks at 168.1 and $169.8 \mathrm{eV}$ corresponding to respective $\mathrm{S} 2 p_{3 / 2}$ and $S 2 p_{1 / 2}$ are observed in the MeS-treated perovskite film ${ }^{49}$. Figure $4 c$, d shows the XPS spectra for $\mathrm{Pb}$ and $\mathrm{Br}$, respectively, where for the photoemission of $\mathrm{Pb} 4 f$, both $\mathrm{Pb} 4 f_{7 / 2}(138.2 \mathrm{eV})$ and $\mathrm{Pb} 4 f_{5 / 2}$ $(142.9 \mathrm{eV})$, signals in the $8 \%-\mathrm{MeS}$-treated film shift to lower binding energy by around $0.2 \mathrm{eV}$. Such negative shifts of $\mathrm{Pb} 4 f$ binding energy indicate the decrease of the cationic charge on the $\mathrm{Pb}$ ion since the $\mathrm{S}=\mathrm{O}$ donates the lone electron pair on the oxygen atoms to the empty $6 p$ orbital of $\mathrm{Pb}^{2+}$ cations ${ }^{49}$. The shift toward lower binding energy is also observed for the $\mathrm{Br} 3 d$ spectrum, which is caused by the disturbance due to the electron donation from $\mathrm{MeS}$ to $\mathrm{Pb}^{2+}$ through the formation of Lewis adducts, subsequently resulting also in the change of static interactions between $\mathrm{Pb}^{2+}$ and $\mathrm{Br}^{-}$ions ${ }^{50}$. Besides, the strong affinity of BA with $\mathrm{MeS}$ reduces the chance of $\mathrm{BA}$ adsorption on the perovskite surface, and this weakens the blocking effect of BA on the growth of the perovskite. As the blocking effect of the adsorbate tends to induce defects in crystal growth ${ }^{51-53}$, the reduced BA adsorption caused by the presence of MeS may also contribute to alleviating defect formation in the perovskite films.

To support this hypothesis, the defect density was evaluated for hole-only devices with the structures of ITO/TFB:PVK/ $\mathrm{PVP} /$ perovskite $/ \mathrm{MoO}_{3} / \mathrm{Al}$ by performing space-charge-limited current (SCLC) measurements (Supplementary Fig. 9). The device current has a linear proportionality to the drive voltage up to the trap-filled limit $\left(V_{\mathrm{TFL}}\right)^{54-56}$. It can be seen that the MeS-treated film presented a lower $V_{\mathrm{TFL}}(1.08 \mathrm{~V})$ compared to those of the control film $(1.45 \mathrm{~V})$, demonstrating that the trap density in the perovskite film was reduced. Consequently, we extracted a trap density of $n_{\text {traps }}=(5.05 \pm 0.18) \times 10^{17} \mathrm{~cm}^{-3}$ for the MeS-treated film, which is lower than that of the control film $\left(n_{\text {traps }}=(9.87 \pm 0.45) \times 10^{17} \mathrm{~cm}^{-3}\right)$. We further observed that, when the perovskite films were excited under different excitation powers, both the PLQYs of the control and MeStreated films increased with the increase of excitation power, and the MeS-treated films showed remarkably higher PLQY values over the whole excitation range (Supplementary Fig. 10) due to the increased fraction of bimolecular recombination and trap-filling behavior ${ }^{17}$. 
a

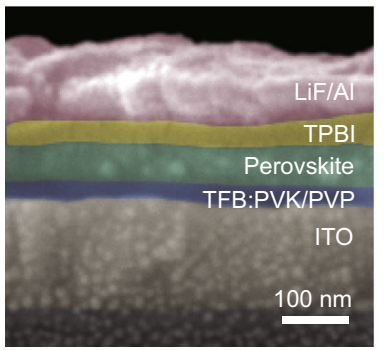

d

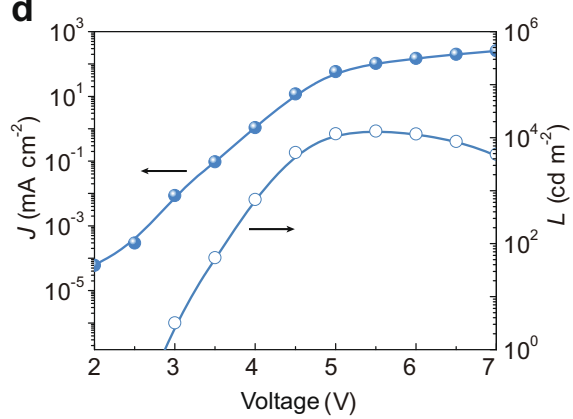

b

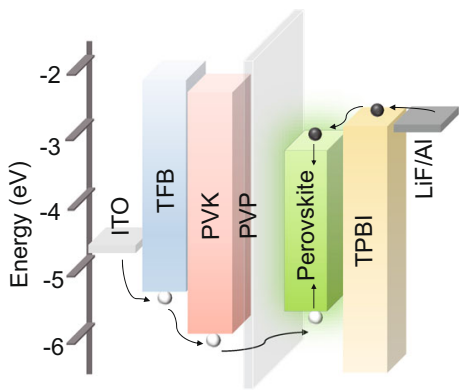

e

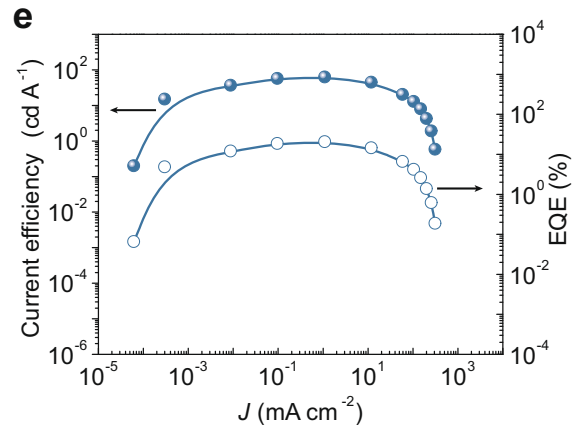

C

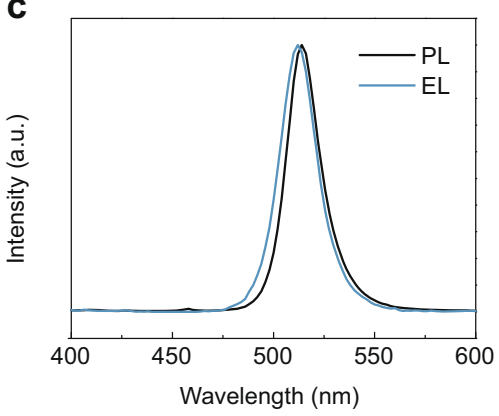

f

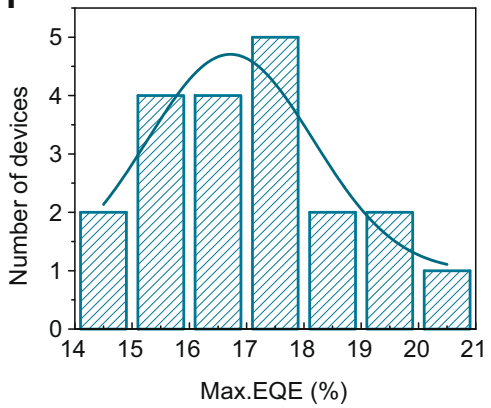

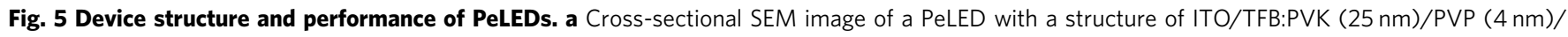
perovskite $(50 \mathrm{~nm}) / \mathrm{TPBI}(35 \mathrm{~nm}) / \mathrm{LiF}(1 \mathrm{~nm}) / \mathrm{Al}(100 \mathrm{~nm})$, and $\mathbf{b}$ its schematic energy band diagram. Except for the perovskite layer which is directly

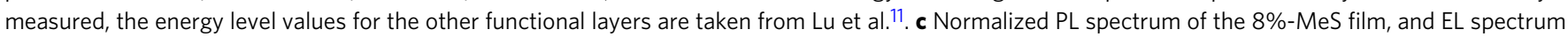

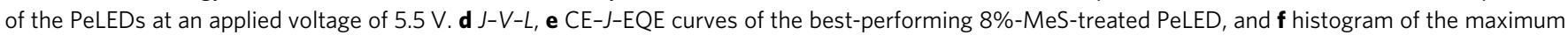
EQEs measured on 20 such devices.

Exciton energy transfer manipulation for efficient PeLEDs. Green PeLEDs with structures of ITO/TFB:PVK/control perovskite/1,3,5-tris(1-phenyl-1H-benzimidazol-2-yl)benzene (TPBI)/lithium fluoride (LiF)/aluminum (Al) (Device A), ITO/ TFB:PVK/MeS-treated perovskite/TPBI/LiF/Al (Device B), ITO/ TFB:PVK/PVP/MeS-treated perovskite/TPBI/LiF/Al (Device C), and ITO/TFB:PVK/PVP/control perovskite/TPBI/LiF/Al (Device D) have been fabricated and compared. In the cross-sectional scanning electron microscopy (SEM) image (Fig. 5a), each of the functional layers in Device C (except for the ultrathin, $\sim 4 \mathrm{~nm}$ PVP film) can be clearly seen. The device energy band diagram is plotted in Fig. 5b, based on energy level values for this perovskite film as obtained by ultraviolet photoelectron spectroscopy (UPS) coupled with optical measurements (Supplementary Fig. 11). Note that the presence of an ultrathin layer of PVP on top of the TFB:PVK mixed HTL plays an important role in improving film quality and ensuing better device performance (Supplementary Fig. 12). It not only enables the deposition of smooth and dense perovskite films due to the significantly improved HTL surface wettability (Supplementary Fig. 13), but also suppresses the emission quenching by suppressing charge transfer at the perovskite/HTL interface ${ }^{57,58}$ (Supplementary Fig. 14). In the absence of PVP (in both cases), Device B shows an almost twofold performance enhancement compared to the Device A (13.14\% EQE \& $10,140 \mathrm{~cd} \mathrm{~m}^{-2}$ luminance vs. $6.37 \%$ EQE \& $6094 \mathrm{~cd} \mathrm{~m}^{-2}$ luminance, respectively. Supplementary Fig. 15 and Supplementary Table 3).

Figure $5 c-f$ shows the performance characteristics of our best green PeLED with MeS-treated perovskite and PVP interlayer (Device C). The EL spectrum matches well with the PL spectrum (Fig. 5c), and the EL emission peak (at $512 \mathrm{~nm}$ ) is only slightly blueshifted and broadened. The blueshift most probably results from free carrier emission, as already demonstrated in several perovskite systems ${ }^{59-61}$; and the broadened EL can be attributed to the electric-field-induced Stark effect ${ }^{2,62}$. From the current density-luminance-voltage $(J-V-L)$ curves (Fig. $5 \mathrm{~d}$ ), we can see that the current density rapidly increased after reaching the turnon voltage $(\sim 2.8 \mathrm{~V})$ due to efficient carrier injection into the perovskite emission layer. As the current density continued to increase, the device had a peak luminance of $13,400 \mathrm{~cd} \mathrm{~m}^{-2}$ at a low applied voltage of $5.5 \mathrm{~V}$, and a high $\mathrm{CE}$ of $63 \mathrm{~cd} \mathrm{~A}^{-1}$ corresponding to an EQE of $20.5 \%$ at a current density of $\sim 1$ $\mathrm{mA} \mathrm{cm}{ }^{-2}$, which is, to the best of our knowledge, a record efficiency in quasi-2D PeLEDs for all colors (Supplementary Table 4 compares our results with recently reported values in the literature). A histogram of the maximum EQE values for 20 devices (Fig. 5f) shows an average EQE of $17.1 \%$ with a relative standard deviation of $10 \%$, indicating good device-to-device reproducibility. Furthermore, due to the reduced ion migration pathways in the MeS-treated perovskite films, the device stability becomes remarkably improved as well, so that the Device C shows a two-fold operational lifetime enhancement compared with the Device D (Supplementary Fig. 16).

\section{Discussion}

We achieved a record efficiency (CE of $63 \mathrm{~cd} \mathrm{~A}^{-1}$ and EQE of $20.5 \%)$ for quasi-2D BA $\mathrm{Bs}_{n-1} \mathrm{~Pb}_{n} \mathrm{Br}_{3 n+1}$ PeLEDs by introducing $\mathrm{MeS}$ into the perovskite precursor solutions to reconstruct the perovskite phase distribution and reduce uncoordinated $\mathrm{Pb}^{2+}$ ions, and thus enhance the emission efficiency of perovskite films derived from these solutions. The proportion of large- $n$ phases with narrow bandgap has increased in the distribution phases of different $n$ values of quasi-2D perovskites due to hydrogenbonding interaction between $-\mathrm{SO}_{3}{ }^{-}$group of $\mathrm{MeS}$ and $\mathrm{BA}$ spacer; and the surface uncoordinated $\mathrm{Pb}^{2+}$ ions on the perovskites were simultaneously reduced benefiting from the electron-rich Lewis base nature of MeS. Besides, the blocking effect of BA which tends 
to induce defects during crystal growth could be reduced because of the interaction between BA and MeS. This work offers a promising approach for fabricating next-generation high-performance PeLEDs based on quasi-2D perovskites.

\section{Methods}

Materials. $\mathrm{CsBr}$ (99.999\%, metal basis) and $\mathrm{PbBr}_{2}$ (99.999\%, metal basis) were purchased from Alfa Aesar. BABr was purchased from Xi'an Polymer Light Technology Corp. CsMeS, PVK, PVP, PEO, and DMSO (99.9\%) were purchased from Sigma-Aldrich. TFB, TPBI, and LiF were purchased from Luminescence Technology Corp. Absolute ethyl alcohol and toluene were purchased from Sinopharm Chemical Reagent Co., Ltd.

Perovskite precursor solutions. $\mathrm{PbBr}_{2}, \mathrm{CsBr}$, and $\mathrm{BABr}$ were dissolved in anhydrous DMSO with the ratio of 1:1.2:0.4, and PEO $\left(3.33 \mathrm{mg} \mathrm{m}^{-1}\right)$ was added to improve the morphology of the resulting film ${ }^{63-65}$. CsMeS was added into the precursor solution, with its molar ratios to $\mathrm{PbBr}_{2}$ being $6 \%, 8 \%$, and $10 \%$, respectively. The mixture was stirred at $60^{\circ} \mathrm{C}$ overnight and filtered through a $0.45 \mu \mathrm{m}$ polytetrafluoroethylene membrane before using.

Device Fabrication. ITO substrates were sonicated in sequence with detergent, deionized water, acetone, and isopropyl alcohol for $20 \mathrm{~min}$, respectively, and then dried under nitrogen flow. TFB and PVK were mixed in a weight ratio of 4:6 and dissolved in chlorobenzene $\left(10 \mathrm{mg} \mathrm{ml}^{-1}\right)$. A TFB:PVK layer was spin-coated onto oxygen-plasma-treated ITO substrates at $4000 \mathrm{rpm}$ for $40 \mathrm{~s}$, followed by annealing at $150^{\circ} \mathrm{C}$ for $20 \mathrm{~min}$. PVP $\left(1.5 \mathrm{mg} \mathrm{ml}^{-1}\right.$ in absolute ethyl alcohol $)$ was spin-coated on top of the TFB:PVK layer. For the perovskite film, the precursor solution was spin-coated at $4000 \mathrm{rpm}$ for $50 \mathrm{~s}$-after spin coating for $40 \mathrm{~s}, 100 \mu \mathrm{l}$ toluene antisolvent was dropped onto the film and spin coating continued for a further $10 \mathrm{~s}-$ followed by baking at $80^{\circ} \mathrm{C}$ for $10 \mathrm{~min}$. After that, TPBI $(40 \mathrm{~nm}), \operatorname{LiF}(1 \mathrm{~nm})$, and $\mathrm{Al}$ electrode $(100 \mathrm{~nm})$ layers were sequentially deposited by thermal evaporation under a vacuum of $\sim 4 \times 10^{-4} \mathrm{~Pa}$.

Characterization. XRD patterns were collected on a Bruker D8 Advance diffractometer with $\mathrm{Cu} \mathrm{Ka}$ radiation as the $\mathrm{X}$-ray source, ranging from $5^{\circ}$ to $60^{\circ}$ at a scanning rate of $6^{\circ} \mathrm{min}^{-1}$. UV-Vis spectra were recorded on a Perkin Elmer Lambda 950 UV-Vis-NIR spectrometer. TA measurements were carried out on a Helios pump-probe system (Ultrafast Systems LLC) with a 365-nm laser $(0.17$ $\mathrm{mW})$. XPS and UPS spectra were acquired using a Thermo Scientific Escalab 250Xi. He (I) ultraviolet radiation source $(21.22 \mathrm{eV})$ from a He discharge lamp was used in the UPS measurements. The work functions (WFs) and the highest occupied molecular orbital (HOMO) regions were extracted from the UPS spectra using the equations $\mathrm{WF}=21.22-E_{\text {cutoff }}$ and $\mathrm{HOMO}=\mathrm{WF}+E_{\text {onset }}$. The $E_{\text {cutoff }}$ and the $E_{\text {onet }}$ were determined by the intercepts of the tangents of the peaks with the extrapolated baselines, as depicted in the relevant plots. Steady-state PL spectra and TRPL were recorded on an Edinburgh FLS920 PL spectrometer at an excitation wavelength of $365 \mathrm{~nm} .{ }^{1} \mathrm{H}$ NMR spectra were recorded on a Bruker Advanced II $(400 \mathrm{MHz})$ spectrometer at room temperature. The grazing-incidence wide-angle X-ray scattering (GIWAXS) data were obtained at beamline BL14B1 of the Shanghai Synchrotron Radiation Facility (SSRF), China. A monochromatic beam of $\lambda=0.6887 \AA$ was used, and the incident angle was $0.1^{\circ}$. The EL characteristics of the PeLEDs were collected on a system comprised of a PR-670 Spectra Scan Spectroradiometer coupled with a Keithley 2400 sourcemeter. The EQEs were calculated from the luminance, the current density, and the EL emission spectra of devices. All the device characterization tests were carried out on unencapsulated PeLEDs at room temperature in ambient air, except that the operational stability test was carried in an $\mathrm{N}_{2}$-filled glovebox.

DFT simulations. The reaction Gibbs free energy $\Delta G$ for the reaction, CsMeS + $\mathrm{BABr}=\mathrm{BAMeS}+\mathrm{CsBr}$, was approximated by the change of ground-state energy at $0 \mathrm{~K}: \Delta G \approx E_{\mathrm{BAMeS}}+E_{\mathrm{CsBr}}-E_{\mathrm{BABr}}-E_{\mathrm{CsMes}}$. The ground-state energy was calculated using the Vienna Ab Initio Simulations Package (VASP) and projected augmented wave (PAW) method with $1 s^{1}, 2 s^{2} 2 p^{2}, 2 s^{2} 2 p^{3}, 2 s^{2} 2 p^{4}, 3 s^{2} 3 p^{4}, 4 s^{2} 4 p^{5}$, $5 s^{2} 5 p^{6} 6 s^{1}$, and $6 s^{2} 6 p^{2}$ as valence electrons for $\mathrm{H}, \mathrm{C}, \mathrm{N}, \mathrm{O}, \mathrm{S}, \mathrm{Br}, \mathrm{Cs}$, and $\mathrm{Pb}$, respectively. The exchange-correlation interaction was treated with the generalized gradient approximation (GGA) parameterized by a Perdew, Burke, and Ernzerhof (PBE) functional. The Brillouin zone was sampled by using a Gamma point for molecules and a Gamma-cantered k-mesh of $9 \times 9 \times 9$ for CsBr. The cutoff energy of the plane-wave basis was chosen to converge the energy to $1 \mathrm{meV}^{\text {atom }}{ }^{-1}$. The force was converged to less than $0.01 \mathrm{eV} \AA^{-1}$ for all structure relaxations. The calculated ground-state energies are $-90.935,-46.738,-133.728$, and $-6.107 \mathrm{eV}$ for $\mathrm{BABr}$, CsMeS, BAMeS, and $\mathrm{CsBr}$, respectively. The reaction free energy is therefore $-2.16 \mathrm{eV}$.

\section{Data availability}

The data that support the plots within this paper and the other findings of this study are available from the corresponding authors upon reasonable request.
Received: 4 September 2020; Accepted: 28 January 2021; Published online: 23 February 2021

\section{References}

1. Liu, Y. et al. Efficient blue light-emitting diodes based on quantum-confined bromide perovskite nanostructures. Nat. Photonics 13, 760-764 (2019).

2. Wang, $\mathrm{H}$. et al. Trifluoroacetate induced small-grained $\mathrm{CsPbBr}_{3}$ perovskite films result in efficient and stable light emitting devices. Nat. Commun. 10, 665 (2019).

3. Kumar, S. et al. Ultrapure green light-emitting diodes using two-dimensional formamidinium perovskites: achieving recommendation 2020 color coordinates. Nano Lett. 17, 5277-5284 (2017).

4. Wang, H. et al. Perovskite-molecule composite thin films for efficient and stable light-emitting diodes. Nat. Commun. 11, 891 (2020).

5. Shi, Z. et al. Uncovering the mechanism behind the improved stability of $2 \mathrm{D}$ organic-inorganic hybrid perovskites. Small 15, 1900462 (2019).

6. Deng, S. et al. Long-range exciton transport and slow annihilation in twodimensional hybrid perovskites. Nat. Commun. 11, 664 (2020).

7. Adjokatse, S., Fang, H.-H. \& Loi, M. A. Broadly tunable metal halide perovskites for solid-state light-emission applications. Mater. Today 20, 413-424 (2017).

8. Lu, J.-H., Yu, Y.-L., Chuang, S.-R., Yeh, C.-H. \& Chen, C.-P. Highperformance, semitransparent, easily tunable vivid colorful perovskite photovoltaics featuring Ag/ITO/Ag microcavity structures. J. Phys. Chem. C 120, 4233-4239 (2016).

9. Wang, Q. et al. Efficient sky-blue perovskite light-emitting diodes via photoluminescence enhancement. Nat. Commun. 10, 5633 (2019).

10. Jiang, Y. et al. Spectra stable blue perovskite light-emitting diodes. Nat. Commun. 10, 1868 (2019)

11. Lu, M. et al. Metal halide perovskite light-emitting devices: promising technology for next-generation displays. Adv. Funct. Mater. 29, 1902008 (2019).

12. Zhao, L., Lee, K. M., Roh, K., Khan, S. U. Z., \& Rand, B. P. Improved outcoupling efficiency and stability of perovskite light-emitting diodes using thin emitting layers. Adv. Mater. 31, 1805836 (2019).

13. Yuan, Z. et al. Unveiling the synergistic effect of precursor stoichiometry and interfacial reactions for perovskite light-emitting diodes. Nat. Commun. 10, 2818 (2019).

14. Cao, Y. et al. Perovskite light-emitting diodes based on spontaneously formed submicrometre-scale structures. Nature 562, 249-253 (2018).

15. Xu, W. et al. Rational molecular passivation for high-performance perovskite light-emitting diodes. Nat. Photonics 13, 418-424 (2019).

16. Lin, K. et al. Perovskite light-emitting diodes with external quantum efficiency exceeding 20 percent. Nature 562, 245-248 (2018).

17. $\mathrm{Wu}, \mathrm{T}$. et al. High-performance perovskite light-emitting diode with enhanced operational stability using lithium halide passivation. Angew. Chem. Int. Ed. 59, 4099-4105 (2020).

18. Shen, H. et al. Visible quantum dot light-emitting diodes with simultaneous high brightness and efficiency. Nat. Photonics 13, 192-197 (2019).

19. Song, J. et al. Over $30 \%$ external quantum efficiency light-emitting diodes by engineering quantum dot-assisted energy level match for hole transport layer. Adv. Funct. Mater. 29, 1808377 (2019).

20. Rajamalli, P. et al. A new molecular design based on thermally activated delayed fluorescence for highly efficient organic light emitting diodes. J. Am. Chem. Soc. 138, 628-634 (2016).

21. Tan, Z.-K. et al. Bright light-emitting diodes based on organometal halide perovskite. Nat. Nanotechnol. 9, 687-692 (2014).

22. Xiao, Z. et al. Efficient perovskite light-emitting diodes featuring nanometresized crystallites. Nat. Photonics 11, 108-115 (2017).

23. Liu, T. et al. Tailoring vertical phase distribution of quasi-two-dimensional perovskite films via surface modification of hole-transporting layer. Nat. Commun. 10, 878 (2019).

24. Zhou, N. et al. Exploration of crystallization kinetics in quasi two-dimensional perovskite and high performance solar cells. J. Am. Chem. Soc. 140, 459-465 (2017).

25. Cheng, L. et al. Multiple-quantum-well perovskites for high-performance light-emitting diodes. Adv. Mater. 32, 1904163 (2019).

26. Lin, Y. et al. Suppressed ion migration in low-dimensional perovskites. ACS Energy Lett. 2, 1571-1572 (2017).

27. $\mathrm{Li}, \mathrm{C}$. et al. Understanding the improvement in the stability of a self-assembled multiple-quantum well perovskite light-emitting diode. J. Phys. Chem. Lett. 10, 6857-6864 (2019).

28. Yang, X. et al. Efficient green light-emitting diodes based on quasi-twodimensional composition and phase engineered perovskite with surface passivation. Nat. Commun. 9, 570 (2018). 
29. Ban, M. et al. Solution-processed perovskite light emitting diodes with efficiency exceeding $15 \%$ through additive-controlled nanostructure tailoring. Nat. Commun. 9, 3892 (2018).

30. Leng, M. et al. Surface passivation of bismuth-based perovskite variant quantum dots to achieve efficient blue emission. Nano Lett. 18, 6076-6083 (2018).

31. Yin, W.-J., Yang, J.-H., Kang, J., Yan, Y. \& Wei, S.-H. Halide perovskite materials for solar cells: a theoretical review. J. Mater. Chem. A 3, 8926-8942 (2015).

32. Kim, J., Chung, C.-H. \& Hong, K.-H. Understanding of the formation of shallow level defects from the intrinsic defects of lead tri-halide perovskites. Phys. Chem. Chem. Phys. 18, 27143-27147 (2016).

33. Sapori, D. et al. Quantum confinement and dielectric profiles of colloidal nanoplatelets of halide inorganic and hybrid organic-inorganic perovskites. Nanoscale 8, 6369-6378 (2016).

34. Xia, P. et al. A pre-solution mixing precursor method for improving crystallization quality of perovskite film and electroluminescent performance of perovskite light-emitting diodes. Nanoscale 11, 20847-20856 (2019).

35. Quan, L. N. et al. Ligand-stabilized reduced-dimensionality perovskites. J. Am. Chen. Soc. 138, 2649-2655 (2016).

36. $\mathrm{Du}$, J. et al. Ionic liquid-assisted improvements in the thermal stability of $\mathrm{CH}_{3} \mathrm{NH}_{3} \mathrm{PbI}_{3}$ perovskite photovoltaics. Phys. Status Solidi RRL 12, 1800130 (2018).

37. Yuan, S. et al. Optimization of low-dimensional components of quasi-2D perovskite films for deep-blue light-emitting diodes. Adv. Mater. 31, 1904319 (2019).

38. Quan, L. N. et al. Tailoring the energy landscape in quasi-2D halide perovskites enables efficient green-light emission. Nano Lett. 17, 3701-3709 (2017).

39. Yuan, M. et al. Perovskite energy funnels for efficient light-emitting diodes. Nat. Nanotechnol. 11, 872-877 (2016).

40. Liu, J., Leng, J., Wu, K., Zhang, J. \& Jin, S. Observation of internal photoinduced electron and hole separation in hybrid two-dimensional perovskite films. J. Am. Chem. Soc. 139, 1432-1435 (2017).

41. Cohen, B.-E., Wierzbowska, M. \& Etgar, L. High efficiency quasi 2D lead bromide perovskite solar cells using various barrier molecules. Sustain. Energy Fuels 1, 1935-1943 (2017).

42. Lei, L. et al. Effcient energy funneling in quasi-2D perovskites: from light emission to lasing. Adv. Mater. 32, 1906571 (2020).

43. Cho, H. et al. Overcoming the electroluminescence efficiency limitations of perovskite light-emitting diodes. Science 350, 1222-1225 (2015).

44. Ren, Z. et al. Hole transport bilayer structure for quasi-2D perovskite based blue light-emitting diodes with high brightness and good spectral stability. Adv. Funct. Mater. 29, 1905339 (2019).

45. Xing, G. et al. Transcending the slow bimolecular recombination in leadhalide perovskites for electroluminescence. Nat. Commun. 8, 14558 (2017).

46. Zhou, W. et al. Zwitterion coordination induced highly orientational order of $\mathrm{CH}_{3} \mathrm{NH}_{3} \mathrm{PbI}_{3}$ perovskite film delivers a high open circuit voltage exceeding 1.2 V. Adv. Funct. Mater. 29, 1901026 (2019).

47. Wang, Q. et al. Stabilizing the a-phase of $\mathrm{CsPbI}_{3}$ perovskite by sulfobetaine zwitterions in one-step spin-coating films. Joule 1, 371-382 (2017).

48. Deepa, M., Sharma, N., Agnihotry, S. A. \& Chandra, R. FTIR investigations on ion-ion interactions in liquid and gel polymeric electrolytes: $\mathrm{LiCF}_{3} \mathrm{SO}_{3}-\mathrm{PC}$ PMMA. J. Mater. Sci. 37, 1759-1765 (2002).

49. Han, M. G. \& Im, S. S. X-ray photoelectron spectroscopy study of electrically conducting polyaniline/polyimide blends. Polymer 41, 3253-3262 (2000).

50. Li, B. et al. Anchoring fullerene onto perovskite film via grafting pyridine toward enhanced electron transport in high-efficiency solar cells. ACS Appl. Mater. Interfaces 10, 32471-32482 (2018).

51. Chen, D., Gallagher, M. J. \& Sarid, D. Scanning tunneling microscopy study of the adsorption of C60 molecules on Si (100)- $(2 \times 1)$ surfaces. J. Vac. Sci. Technol. B 12, 1947-1951 (1994).

52. Taubert, A., Kübel, C. \& Martin, D. C. Polymer-induced microstructure variation in zinc oxide crystals precipitated from aqueous solution. J. Phys. Chem. B 107, 2660-2666 (2003).

53. Duan, C. et al. Engineering new defects in MIL-100 (Fe) via a mixed-ligand approach to effect enhanced volatile organic compound adsorption capacity. Ind. Eng. Chem. Res. 59, 774-782 (2019).

54. Shen, $\mathrm{X}$. et al. Zn-alloyed $\mathrm{CsPbI}_{3}$ nanocrystals for highly efficient perovskite light-emitting devices. Nano Lett. 19, 1552-1559 (2019).

55. $\mathrm{Wu}, \mathrm{X}$. et al. Stable triple cation perovskite precursor for highly efficient perovskite solar cells enabled by interaction with $18 \mathrm{C} 6$ stabilizer. Adv. Funct. Mater. 30, 1908613 (2020).

56. Wang, H. et al. Molecule-induced p-doping in perovskite nanocrystals enables efficient color-saturated red light-emitting diodes. Small 16, 2001062 (2020).
57. Zhang, X. et al. Enhancing the brightness of cesium lead halide perovskite nanocrystal based green light-emitting devices through the interface engineering with perfluorinated ionomer. Nano Lett. 16, 1415-1420 (2016).

58. Pal, B. N. et al. 'Giant' CdSe/CdS core/shell nanocrystal quantum dots as efficient electroluminescent materials: strong influence of shell thickness on light-emitting diode performance. Nano Lett. 12, 331-336 (2012).

59. Zhang, L. et al. Ultra-bright and highly efficient inorganic based perovskite light-emitting diodes. Nat. Commun. 8, 15640 (2017).

60. Palma, A. L. et al. Mesoscopic perovskite light-emitting diodes. ACS Appl. Mater. Interfaces 8, 26989-26997 (2016).

61. D'Innocenzo, V. et al. Excitons versus free charges in organo-lead tri-halide perovskites. Nat. Commun. 5, 3586 (2014).

62. Yang, X. et al. Electroluminescence efficiency enhancement in quantum dot light-emitting diodes by embedding a silver nanoisland layer. Adv. Opt. Mater. 3, 1439-1445 (2015).

63. Jeong, B. et al. All-inorganic $\mathrm{CsPbI}_{3}$ perovskite phase-stabilized by poly (ethylene oxide) for red-light-emitting diodes. Adv. Funct. Mater. 28, 1706401 (2018).

64. Tian, Y. et al. Highly efficient spectrally stable red perovskite light-emitting diodes. Adv. Mater. 30, 1707093 (2018).

65. Wu, C. et al. Improved performance and stability of all-inorganic perovskite light-emitting diodes by antisolvent vapor treatment. Adv. Funct. Mater. 27, 1700338 (2017).

\section{Acknowledgements}

We acknowledge financial support from the National Natural Science Foundation of China (Nos. 51675322, 61605109, 61735004, 51702115, and 51972136), the National Key Research and Development Program of China (No. 2016YFB0401702), and the Shanghai Science and Technology Committee (No. 19010500600). We thank the staff from BL01B1, BL11B, BL17B1 and BL19U1 beamlines of Shanghai Synchrotron Radiation Facility for assistance during data collection. A.R. also thanks the financial support from the Research Grant Council of Hong Kong (CRF C7035-20G) and the Croucher Foundation of Hong Kong.

\section{Author contributions}

X.Y. and A.L.R. designed and directed the study. L.K. and H.W. contributed to LED device fabrication. Y.J. carried out the TA measurements and analysis. Y.L. performed the DFT simulations. L.K., X.Z., Y.L., H.W., S.W., M.Y., C.Z., T.Z., S.V.K., W.Z., Y.Y., Q.L., M.Y., A.L.R., and X.Y. analyzed and discussed the experimental results. L.K., X.Z., and Y.L. contributed equally to this work. All authors contributed to writing the manuscript.

\section{Competing interests}

The authors declare no competing interests.

\section{Additional information}

Supplementary information The online version contains supplementary material available at https://doi.org/10.1038/s41467-021-21522-8.

Correspondence and requests for materials should be addressed to A.L.R. or X.Y.

Peer review information Nature Communications thanks Jianpu Wang and the other, anonymous, reviewer(s) for their contribution to the peer review of this work.

Reprints and permission information is available at http://www.nature.com/reprints

Publisher's note Springer Nature remains neutral with regard to jurisdictional claims in published maps and institutional affiliations.

\footnotetext{
(c) (i) Open Access This article is licensed under a Creative Commons Attribution 4.0 International License, which permits use, sharing, adaptation, distribution and reproduction in any medium or format, as long as you give appropriate credit to the original author(s) and the source, provide a link to the Creative Commons license, and indicate if changes were made. The images or other third party material in this article are included in the article's Creative Commons license, unless indicated otherwise in a credit line to the material. If material is not included in the article's Creative Commons license and your intended use is not permitted by statutory regulation or exceeds the permitted use, you will need to obtain permission directly from the copyright holder. To view a copy of this license, visit http://creativecommons.org/ licenses/by/4.0/.
}

(c) The Author(s) 2021 